\title{
Rhabdomyolysis After Ankle Strain and Light Cycling
}

\author{
James $\mathrm{Hu}^{\mathrm{a}, \mathrm{c}}$, David $\mathrm{Ng}^{\mathrm{b}}$
}

\begin{abstract}
A 35-year-old female presented to the emergency room with severe upper leg and back pain, which began 3 days after low-intensity cycling and falling from a stationary bike. She developed rhabdomyolysis with a maximum serum creatine kinase level of 72,358 U/L. This case report demonstrates that rhabdomyolysis has a wide range and spectrum of causes and risk factors. Although uncommon, this condition can occur after low-intensity exercise despite absence of other significant risk factors. Thus, clinicians should maintain a high clinical suspicion when initial history, physical examination, and laboratory tests suggest this diagnosis.
\end{abstract}

Keywords: Rhabdomyolysis; Spinning machine; Cycling; Low-intensity exercise; Gym

\section{Introduction}

Rhabdomyolysis is a condition in which intramuscular contents are released into the bloodstream as a result of muscle necrosis [1]. It is characterized by elevation of creatine kinase (CK), muscle pain, and/or myoglobinuria. These effects of muscle necrosis can result in a range of conditions: from asymptomatic to severe kidney injury [2]. A proposed mechanism of kidney injury is due to excess myoglobin, leading to primary nephrotoxicity, which results in tubular obstruction, oxidant injury, and vasoconstriction [3]. Etiology includes crush injuries, prolonged immobilization, intense physical exertion, infections, electrolyte imbalance, drug/toxins, etc. Symptoms often include muscle ache, dark urine, weakness;

Manuscript accepted for publication July 01, 2016

aDepartment of Family Medicine, Nassau University Medical Center, 161 Hempstead Turnpike, Elmont, NY 11003, USA

bepartment of Emergency Medicine, Nassau University Medical Center, 2201 Hempstead Turnpike, East Meadow, NY 11554, USA

${ }^{\mathrm{c} C}$ Corresponding Author: James Hu, Department of Family Medicine, Nassau University Medical Center, 161 Hempstead Turnpike, Elmont, NY 11003, USA.Email: jhu1@numc.edu

doi: http://dx.doi.org/10.14740/jocmr2626w patients often present with body and muscle tenderness [2] However, the hallmark for diagnosing rhabdomyolysis is the elevation of serum CK level.

After muscle injury, serum CK level usually begins to rise between 2 and $12 \mathrm{~h}$ window, reaches maximum level within 48 $-72 \mathrm{~h}$, then begins to decline 3 - 5 days after injury. The CK value is usually five times or more of the normal serum level, but can range from 1,500 to $100,000+$ IU [1]. Other manifestations may include electrolyte abnormalities, fluid imbalance, kidney injury, and/or compartment syndrome. The most important management of rhabdomyolysis is immediate diuresis/hydration with either normal saline or half-normal saline, followed by close monitoring of renal function and electrolytes [1].

In the past, there had been reports of patients developing rhabdomyolysis from vigorous stationary cycling sessions, especially in first-timers or those who are physically untrained for such intensity $[4,5]$. However, below is a case of unexpected rhabdomyolysis from relatively low-intensity activity with benign injuries in an otherwise healthy individual.

\section{Case Report}

\section{History}

A 35-year-old Hispanic female with no past medical history and no home medication, presented to the emergency room complaining of severe bilateral lower extremity pain and tightness. Three days prior to her emergency department (ED) visit, she fell from a cycling machine at the gym due to handle malfunction, twisted her ankle, and landed on her knees. However, she sustained no external injury and continued to ride the stationary bike lightly for another $45 \mathrm{~min}$. After returning home, patient was able to ambulate and conduct her daily activities despite mild pain and soreness in her knees. After 2 days, on the night prior to coming to the ED, patient began to experience severe pain in both legs, which kept her up all night. She took over-the-counter ibuprofen which provided minimal relief. Next morning, she drove herself to the emergency room. In the ED, she reported an 8/10 burning pain and tightness in her lower extremities, especially in her anterior thighs and in her left posterior thigh. She also complained of tingling sensation in her calves bilaterally.

Patient denied having sustained any open or external in- 
Table 1. Complete Blood Count Lab Result on Admission

\begin{tabular}{ll}
\hline Complete blood count & \\
\hline WBC & $5,150 / \mathrm{mm}^{3}$ \\
$\mathrm{Hgb}$ & $11.8 \mathrm{~g} / \mathrm{dL}$ \\
$\mathrm{Hct}$ & $35 \%$ \\
$\mathrm{Plt}$ & $249,000 / \mathrm{mm}^{3}$ \\
\hline
\end{tabular}

jury, but complained of moderate lower legs swelling with severe pain with ambulation or weight-bearing. She had decreased sensation in her lower extremities and tingling sensation in the left leg. Patient also reported lower back pain since the incident, but denied direct injury or trauma to the area. She also denied having any neck pain, headache, fever, chills, shortness of breath, nausea, or vomiting.

Past surgical history: C-section; allergy: no known drug allergy; social Hx: occasional alcohol use, non-smoker, no recreational drug use; family $\mathrm{Hx}$ : non-contributory.

\section{Physical exam}

Vitals: BP, 125/81; HR, 86/min; RR, 19/min; temperature, 97.7 ${ }^{\circ} \mathrm{F}$. General: moderate distress from pain, alert, A\&O $\times 3$, cooperative. HEENT: pupils equal round reactive to light with accommodation, extraocular muscles intact bilaterally. Cardiac: S1S2, regular rate and rhythm, no murmur. Lung: clear to auscultation bilaterally, no wheezes/rales/rhonchi. Abdomen: LLQ tender, normal bowel sound, no rebound/guarding. Back: non-tender, non-traumatic. Skin: intact with no discoloration, rash, or erythema in the lower extremities and lower back. Extremity: bilateral feet - no swelling, non-tender, pedal pulse strong and equal bilaterally; lower legs - mild swelling, paresthesia in calves, non-erythematous; bilateral knees - tender, swelling, limited range of motion due to pain, stable joint, intact skin, no erythema; thighs and hips - bilateral tenderness

Table 2. Comprehensive Metabolic Panel Lab Result on Admission

\begin{tabular}{ll}
\hline Comprehensive metabolic panel & \\
\hline $\mathrm{Na}$ & $140 \mathrm{mmol} / \mathrm{L}$ \\
$\mathrm{Cl}$ & $108 \mathrm{mmol} / \mathrm{L}$ \\
$\mathrm{K}$ & $3.7 \mathrm{mmol} / \mathrm{L}$ \\
$\mathrm{Bicarb}$ & $23 \mathrm{mmol} / \mathrm{L}$ \\
$\mathrm{Cr}$ & $0.4 \mathrm{mg} / \mathrm{dL}$ \\
$\mathrm{BUN}$ & $6 \mathrm{mg} / \mathrm{dL}$ \\
Glucose & $87 \mathrm{mg} / \mathrm{dL}$ \\
AST & $131 \mathrm{U} / \mathrm{L}$ \\
ALT & $56 \mathrm{U} / \mathrm{L}$ \\
ALP & $82 \mathrm{U} / \mathrm{L}$ \\
Creatine kinase & $6,812 \mathrm{U} / \mathrm{L}$ \\
Lactate & $0.8 \mathrm{mmol} / \mathrm{L}$ \\
\hline
\end{tabular}

Table 3. Urinalysis Lab Result on Admission

\begin{tabular}{ll}
\hline Urinalysis & \\
\hline Leukocyte esterase & Trace \\
Blood & Small \\
Ketone & Negative \\
Nitrite & Negative \\
Clarity & Cloudy \\
Bacteria & Few/HPF \\
RBC & $0-3 / \mathrm{HPF}$ \\
Urine epithelium & $6-10 / \mathrm{HPF}$ \\
Hyaline cast & $0-6 / \mathrm{HPF}$ \\
WBC & $11-20 / \mathrm{HPF}$ \\
\hline
\end{tabular}

with limited hip ROM due to pain, patient was unable to lift leg due to pain, non-erythematous, no swelling. Normal gait but ambulation was limited by pain.

\section{Labs and imaging}

Pertinent labs on admission are shown in Tables 1-3.

X-ray, CT, MRI, and Doppler ultrasound showed no fracture or dislocation in her extremities, no deep vein thrombosis (DVT) and/or spine/disc abnormality.

CT of lumbar spine: no significant finding; MRI of lumbosacral spine: no significant finding; lower extremity venous Doppler: no evidence of DVT in bilateral lower extremities; ultrasound of abdomen: echogenic hepatic parenchyma, commonly due to diffuse fatty infiltration of liver; X-ray bilateral femurs and knees: no acute fracture or dislocation.

\section{Hospital course}

Patient was admitted to inpatient medicine service for rhabdomyolysis management. In her initial evaluation in the emergency room, her laboratory tests were significant for anemia with hemoglobin of $11.8 \mathrm{~g} / \mathrm{dL}$ (Table 1), CK level of 6,812 U/L and elevated AST and ALT (Table 2), and a concurrent urinary tract infection (UTI) (Table 3). She was given several liters of normal saline IV fluid boluses, and continued to receive IV fluid on the inpatient medicine floor. Surgery and neurosurgery services ruled out compartment syndrome and cord compression. Her CK level trended up to a maximum of 72,358 U/L on day 3 of admission. Her pain was controlled by opioids. Renal function remained normal during her hospitalization, although lactate level increased to $2.3 \mathrm{mmol} / \mathrm{L}$ for 1 day and returned to normal range. As for her UTI, she was treated with nitrofurantoin. The patient's transaminitis rose to AST 1,225 U/L and ALT $460 \mathrm{U} / \mathrm{L}$; GI consult service attributed these elevations to rhabdomyolysis. On day 6 of admission, she was free of pain. By day 7, her serum CK decreased to 3,695 U/L and was subsequently discharged home. Patient failed to follow-up at the outpatient clinic and no further information is available after 
hospital discharge.

\section{Discussion}

\section{Pathophysiology}

Causes of rhabdomyolysis are diverse, but the ultimate myocyte destruction and the release of cellular component into circulation are what brings on the disease itself. When ATP becomes depleted inside the muscle cells (e.g. strenuous activities, ischemia), it leads to $\mathrm{Na} / \mathrm{K}$-ATPase and Ca-ATPase pump dysfunction. This increases intracellular ion imbalance (sodium, calcium), results in increased proteolytic enzyme activities and/or osmotic swelling, and ultimately leads to muscle cell death and degradation. Cell contents are then released: potassium, aldolase, phosphate, myoglobin, CK, lactate dehydrogenase, aspartate transaminase, and urate. Besides biochemical disruption of cell membrane, direct damage could also cause myocyte destruction - blunt/crush injury, burn, electrical shock, extreme temperatures, etc. [2].

\section{Risk factors}

A number of medical problems can precipitate rhabdomyolysis: illicit drugs, medication, overdose of substance or medication, viral myositis, alcohol abuse, toxins, trauma, extreme temperatures (heat or cold), muscle ischemia, neuroleptic malignant syndrome, seizure, prolonged immobilization, infection, electrolyte imbalance, endocrine abnormalities (i.e. thyroid disorders), genetic disorders, connective tissue disorders, rheumatologic diseases, biological toxins (i.e. snake venoms, insect bites), and cardiopulmonary resuscitations [6].

Substances commonly involved in rhabdomyolysis may include ethanol, methanol, ethylene glycol, heroin, methadone, barbiturates, cocaine, caffeine, amphetamine, lysergic acid diethylamine, 3,4-methylenedioxy-methamphetamine (street drug Ecstasy or Molly), phencyclidine, benzodiazepine, and toluene (glue sniffing) [7]. However, rhabdomyolysis associated with substance use is often a result of immobilization, physical over-activity, seizure, or ischemia, which are often induced by substance use.

Alcohol can also lead to rhabdomyolysis in several ways, including immobilization after intoxication, direct toxicity to muscle, hypokalemia, and hypophosphatemia. Alcohol withdrawal and seizure should be thus considered when assessing the cause. It is important to keep in mind that patients who are intoxicated, psychotic, agitated, and/or unconscious are also at a higher risk of rhabdomyolysis [3].

When it comes to rhabdomyolysis resulting from physical activities, several risk factors exist and may bring on the condition: physically untrained person, impaired heat loss through sweating (anticholinergic medication, heavy or thick sport equipment), and muscle ischemia as a result of vascular occlusion in sickle cell disease $[8,9]$. Several studies have linked rhabdomyolysis to strenuous exercise in a hot environment. During exercise, potassium is released from muscle cells, which then aids vasodilation and increases blood supply to the area. In a potassium-depleted individual, such as those exercising and sweating, this mechanism becomes limited. The resultant effect is local ischemia and heightened risk of rhabdomyolysis $[10,11]$.

\section{Clinical evaluation}

Diagnosing rhabdomyolysis requires a thorough history, physical, and laboratory testing. It is also very important to recognize the risk factor(s) when interviewing the patient.

Symptoms and physical findings of rhabdomyolysis can range from subclinical to severe. In patients who can verbalize, they often complain of myalgia, limb pain, swelling, edema, muscle stiffness, muscle spasm or cramps, or abdominal pain. They may also report fatigue, nausea/vomiting, fever, red/ brown urine, or weakness. The muscles involved tend to be postural muscles, such as thighs, calves, and lower back.

However, a patient may also present with the manifestation that is consistent with the precipitating cause. For example, a patient with rhabdomyolysis induced by crush injury may present as a trauma case with multiple physical injuries, and a patient with rhabdomyolysis induced by seizure may present as post-ictal. It is imperative to recognize the risk factors and the illnesses associated with this condition.

Laboratory tests should include CK, potassium, phosphorous, calcium, creatinine, BUN/creatinine ratio, anion gap, blood alcohol level, urinalysis, drug screen, and EKG if indicated [3]. AST and ALT may also be included since they both also exist in muscle cells, although ALT is more specific to the liver; AST to ALT ratio may be greater than 2:1 in rhabdomyolysis [12]. These tests can detect the various intracellular components upon myocyte damage, help rule in or rule out the commonly associated etiologies, and monitor downstream pathologic effect of rhabdomyolysis. Clinicians should also monitor for the associated complications, such as acute kidney injury, compartment syndrome, and hyperkalemia.

In the setting outside of hospital or emergency room, where laboratory results cannot be obtained relatively quickly, a urinalysis or serum test can be used to detect myoglobin [13]. However, a key point to remember is to use good clinical judgment when rhabdomyolysis is suspected. For example, a urine dipstick positive for blood/hemoglobin after exercising could be a true rhabdomyolysis, or it could be from hemoglobinuria secondary to mechanical RBC injury in the microvasculature $[14,15]$. Thus, obtaining a thorough history is especially important in ambiguous cases.

This is a case of unexpected rhabdomyolysis from lowintensity injury. There had been reports of rhabdomyolysis that developed after using stationary bikes, but they mostly resulted from high-intensity exercises or occurred in first-time participants going through intense cycling [16]. The classic triggers of rhabdomyolysis like moderate-severe musculoskeletal injuries/ trauma, prolonged inactivity, or high-intensity exercises did not happen in this patient's situation. Rather, the patient's mode of injury was not considered intense at all: she suffered an ankle strain from a minor fall, followed by light exercise. In addition, the patient had no predisposing risk factors for rhabdomyolysis 
- she had no known past medical history, no known metabolic disorder, no prior substance or alcohol use, no use of any dietary supplement, and was not on any home medication.

Although her history placed her as low-risk, her clinical examination pointed towards the diagnosis which was later confirmed by laboratory tests. To further explore factors which may have contributed to her rhabdomyolysis, we can focus on a few points. The foremost is that the patient did fall on her knees and sustained an ankle strain when the stationary bike's handle malfunctioned. She then continued to lightly exercise for $45 \mathrm{~min}$ afterwards. Moreover, this case occurred during July in the US Northeast - when the weather was relatively warm and humid, and she could be dehydrated. After the incident, the patient took over-the-counter ibuprofen to relieve her leg pain. In this situation, she falls into several risk factors discussed above: physical injury, dehydration, physical activities, and medication use.

On the other hand, these points also confound the case in that her injury was minor enough that she exercised for an extended-time period and later ambulated normally at home. Also, the patient did not sustain a fracture or obvious musculoskeletal injury; nor was NSAID a medication commonly associated with rhabdomyolysis. Overall, her rhabdomyolysis was unexpected considering her medical history, the mechanism and extent of her injury, and the intensity of her physical activity.

\section{Conclusion}

Rhabdomyolysis can result from a wide range of causes that lead to myocyte damage. Although it most often occurs from extensive musculoskeletal injury, prolonged immobility, substance or specific medication use, or infection, it can occur though minor injuries and in unsuspecting situations. It is crucial to look at the overall picture when evaluating a patient, as we have all been taught during our medical training. This case serves as a reminder of a disease developed through a less common mechanism of injury, and fortunately was successfully diagnosed and treated.

\section{Conflicts of Interest}

None.

\section{References}

1. Knochel JP. Chapter 11. Rhabdomyolysis. In: Lerma EV,
Berns JS, Nissenson AR. eds. CURRENT Diagnosis \& Treatment: Nephrology \& Hypertension. New York, NY: McGraw-Hill; 2009. http://accessmedicine.mhmedical. com/content.aspx ?bookid $=372 \&$ Sectionid $=39961147$. Accessed May 6, 2016.

2. Chatzizisis YS, Misirli G, Hatzitolios AI, Giannoglou GD. The syndrome of rhabdomyolysis: complications and treatment. Eur J Intern Med. 2008;19(8):568-574.

3. Zimmerman JL, Shen MC. Rhabdomyolysis. Chest. 2013;144(3):1058-1065.

4. Montero J, Lovesio C, Godoy MV, Ruiz G. [Rhabdomyolisis caused by spinning in nine patients]. Medicina (B Aires). 2009;69(1 Pt 2):153-156.

5. Inklebarger J, Galanis N, Kirkos J, Kapetanos G. Exercise-induced rhabdomyolysis from stationary biking: a case report. Hippokratia. 2010;14(4):279-280.

6. Parekh R, Care DA, Tainter CR. Rhabdomyolysis: advances in diagnosis and treatment. Emerg Med Pract. 2012;14(3):1-15; quiz 15.

7. Khan FY. Rhabdomyolysis: a review of the literature. Neth J Med. 2009;67(9):272-283.

8. Ramme AJ, Vira S, Alaia MJ, J VDL, Rothberg RC. Exertional rhabdomyolysis after spinning: case series and review of the literature. J Sports Med Phys Fitness. 2016;56(6):789-793.

9. Quattrone RD, Eichner ER, Beutler A, Adams WB, O'Connor FG. Exercise collapse associated with sickle cell trait (ECAST): case report and literature review. Curr Sports Med Rep. 2015;14(2):110-116.

10. Knochel JP, Schlein EM. On the mechanism of rhabdomyolysis in potassium depletion. J Clin Invest. 1972;51(7):1750-1758.

11. Edwards G, Dora KA, Gardener MJ, Garland CJ, Weston $\mathrm{AH} . \mathrm{K}+$ is an endothelium-derived hyperpolarizing factor in rat arteries. Nature. 1998;396(6708):269-272.

12. Nobre C, Thomas B. Elevation of troponins in rhabdomyolysis. Chest. 2014;145(2):415.

13. Sauret JM, Marinides G, Wang GK. Rhabdomyolysis. Am Fam Physician. 2002;65(5):907-912.

14. Abarbanel J, Benet AE, Lask D, Kimche D. Sports hematuria. J Urol. 1990;143(5):887-890.

15. Oosterom DL, Betjes MG. [Exertion-related abnormalities in the urine]. Ned Tijdschr Geneeskd. 2006;150(11):606610.

16. Hernandez-Contreras ME, Torres-Roca M, HernandezContreras V, Rosa Salazar V, Mar Garcia-Mendez M, Garcia-Perez B, Molina-Boix M. Rhabdomyolysis after initial session of indoor cycling: analysis of 11 patients. J Sports Med Phys Fitness. 2015;55(11):1371-1375. 